Pacific Journal of Mathematics

PROJECTIVE REPRESENTATIONS OF FINITE GROUPS IN

Cr 


\title{
PROJECTIVE REPRESENTATIONS OF FINITE GROUPS IN CYCLOTOMIC FIELDS
}

\author{
HANS OPOLKA
}

It is known that every complex projective representation of a finite group is realizable in a cyclotomic field $\boldsymbol{Q}\left(\xi_{k}\right)$. This note is concerned with the problem of finding the minimal $k$ with this property and gives a partical answer in this direction.

1. Let $G$ be a finite group and let $P: G \rightarrow P G L(n, C)$ be a complex projective representation of $G$. A representative of $P$ is a mapping $T: G \rightarrow G L(n, C)$ such that for all $x \in G$ we have $\pi(T(x))=$ $P(x)$ where $\pi$ denotes the canonical homomorphism $G L(n, C) \rightarrow$ $G L(n, C) / C^{*} \cong P G L(n, C) . \quad P$ is called irreducible if in the vector space $V \cong C^{n}$ there is no proper subspace $W$ such that $T(x) W \subset W$ for all $x \in G . \quad P$ is called realizable in a subfield $K$ of $C$ if there is a function $\alpha: G \rightarrow C^{*}$ and some matrix $A \in G L(n, C)$ such that the coefficients of all the matrices $\alpha(x) A T(x) A^{-1}, x \in G$, belong to $K$. The representative $T$ of $P$ is said to be realizable in a subfield $K$ of $C$ if for some matrix $A \in G L(n, C)$ the coefficients of all the matrices $A T(x) A^{-1}, x \in G$, belong to $K$. A subfield $K$ of $C$ is called a (weak) projective splitting field for $G$ if every (irreducible) projective representation of $G$ is realizable in $K$. For any $k \in N$ denote by $\xi_{k}$ a primitive $k$ th root of unity in $C$. In [6] it is shown that the cyclotomic field $\boldsymbol{Q}\left(\xi_{k}\right), k=|G|$, is a projective splitting field for $G$, and it was asked in the same paper (p. 191) whether $|G|$ could be replaced by the exponent $\exp G$ of $G$. We shall point out an example which gives a negative answer to this question, and then we shall prove the following theorem.

THEOREM. The cyclotomic field $\boldsymbol{Q}\left(\xi_{m}\right), m=\exp G^{\prime} \exp M(G)$, is a weak projective splitting field for $G$.

Here $G^{\prime}$ denotes the commutator subgroup and $M(G)$ the multiplicator of $G$. Note that $\exp G \exp M(G)$ divides $|G|$, comp. [1].

For basic concepts in the theory of projective representations the reader may consult [3], $\mathrm{V}, \S 23-25$.

2. Let $T: G \rightarrow G L(n, C)$ be a representative of the complex projective representation $P: G \rightarrow P G L(n, C)$. Then for all $x, y \in G$ we have $T(x) T(y)=f(x, y) T(x y)$ for some $f(x, y) \in C^{*}$, and the 
mapping $f: G \times G \rightarrow C^{*}$ is a central factor system (2-cocycle). If $P$ is realizable in a subfield $K$ of $C$ we may choose $T$ in such a way that all values of $f$ belong to $K$. Now let $H^{2}\left(G, E^{*}\right)$ be the second cohomology group of the finite group $G$ with respect to the multiplicative group $E^{*}$ of a field $E, E^{*}$ regarded as a trivial $G$-module. $\left(H^{2}\left(G, C^{*}\right)=: M(G)\right.$ is also called "multiplicator".) Any subfield $E \subset C$ contains a primitive $e$ th root of unity, $e$ denoting the exponent of the image of the canonical homomorphism $H^{2}\left(G, E^{*}\right) \rightarrow$ $H^{2}\left(G, C^{*}\right)$ induced by the embedding $E^{*} \subset C^{*}$, comp. [4], (2.1). If $K \subset C$ is a (weak) projective splitting field for $G$, then, by the above argument, the homomorphism $H^{2}\left(G, K^{*}\right) \rightarrow H^{2}\left(G, C^{*}\right)$ is surjective, hence $K$ contains a primitive $\exp M(G)$ th root of unity.

In [2], p. 88, an example of a finite nilpotent group of exponent 4 with multiplicator of exponent 8 is given. Therefore $Q(\sqrt{-1})$ is not a projective splitting field for this group. So the answer to the above mentioned question of Reynolds is negative.

The following example (due to $H$. Pahlings) shows that in general the cyclotomic field $\boldsymbol{Q}\left(\xi_{k}\right), k=\exp M(G)$, is not a (weak) projective splitting field for $G$. Let $D=\left\langle a, b \mid a^{8}=a^{2}=1, b a b=a^{7}\right\rangle$ be the dihedral group of order 16 . It has an irreducible projective representation $P$ with representative $T$ such that

$$
T(a)=\left(\begin{array}{cc}
\varepsilon & 0 \\
0 & \varepsilon^{-1}
\end{array}\right), \quad T(b)=\left(\begin{array}{ll}
0 & 1 \\
1 & 0
\end{array}\right),
$$

$\varepsilon=$ primitive root of unity of order 16 . The factor system $f$, which corresponds to $T$, has order 2. It is known that $\exp M(D)=2$, comp. [3], V, (25.6). But there is no function $\alpha: D \rightarrow C^{*}$ such that $\operatorname{det}(\alpha(a) T(a))=\alpha(\alpha)^{2}$ and $\operatorname{trace}(\alpha(\alpha) T(\alpha))=\alpha(\alpha)(2+\sqrt{2})^{1 / 2} \quad$ both belong to $\boldsymbol{Q}$.

3. Now we shall prove the theorem stated in $\S 1$. A key role is played by Clifford's theory for projective representations, comp. [5], $\$ 2$.

Let $P$ be an irreducible complex projective representation of the finite group $G$ and let $T$ be a representative of $P$ such that the values of the central factor system $f: G \times G \rightarrow C^{*}$ which is determined by the relation $T(x) T(y)=f(x, y) T(x y), x, y \in G$, belong to the group $W$ of $\exp M(G)$ th roots of unity in $C$. Let $\chi$ be the character of $T$, i.e., $\chi(x)=\operatorname{trace}(T(x))$ for all $x \in G$. The restriction $T \mid G^{\prime}$ decomposes as a direct sum of representatives of irreducible projective representations of $G^{\prime}$. Let $S$ be one of them and let $\phi$ be its character. $S$ is realizable in the cyclotomic field $\boldsymbol{Q}\left(\xi_{m}\right), m=$ 
$\exp G^{\prime} \exp M(G)$. This is easily seen as follows. Consider the group $G^{\prime}(f):=\left\{(a, h) \mid a \in W, \quad h \in G^{\prime}\right\}$, the multiplication rule given by $\left(a_{1}, h_{1}\right)\left(a_{2}, h_{2}\right)=\left(a_{1} a_{2} f\left(h_{1}, h_{2}\right), h_{1} h_{2}\right)$. Then lift $S$ to a linear representation $\widetilde{S}$ of $G^{\prime}(f)$ by defining $\widetilde{S}((a, h))$ : $=a S(h)$. It is well known (comp. e.g., [3], V, (19.11)) that $\widetilde{S}$ is realizable in the cyclotomic field $K=\boldsymbol{Q}\left(\xi_{k}\right), k=\exp G^{\prime}(f)$. Therefore this is also true for $S$. But $\exp G^{\prime}(f)$ divides $\exp G^{\prime} \exp M(G)$. Now let $I$ be the inertia group of $\phi$, i.e.,

$$
I=\left\{x \in G \mid\left(f(x, h) f\left(x h, x^{-1}\right) / f\left(x, x^{-1}\right)\right) \phi\left(x h x^{-1}\right)=\dot{\phi}(h) \text { for all } h \in G^{\prime}\right\} .
$$

By Clifford's theory there is a representative $R$ of a projective representation of $I$ such that $T$ is induced by $R$, and the restriction $R \mid G^{\prime}$ decomposes as a direct sum of some copies of $S$. Furthermore, for every $x \in I$ there is an invertible matrix $A^{\prime}(x)$ with coefficients in $K$ such that $A^{\prime}(x) S(h) A^{\prime}(x)^{-1}=S^{x}(h)$ for all $h \in G^{\prime}$. Schur's lemma yields a central factor system $t^{\prime}: I \times I \rightarrow K^{*}$ such that $A^{\prime}(x) A^{\prime}(y)=t^{\prime}(x, y) A^{\prime}(x y)$ for all $x, y \in I$. Choose a set $\left\{u_{\hat{o}}\right\}$ of right coset representatives of $G^{\prime}$ in $I$ and define

$$
A\left(h u_{\hat{o}}\right):=f\left(h, u_{\hat{o}}\right)^{-1} S(h) A^{\prime}\left(u_{\hat{o}}\right), A(h):=S(h)
$$

for all $h \in G^{\prime}$. Then $A$ is a representative of a projective representation of $I$ which is realizable in $K$. Denote by $t: I \times I \rightarrow K^{*}$ the central factor system which belongs to $A$. The central factor system $f t^{-1}: I \times I \rightarrow K^{*}$ satisfies $f t^{-1}\left(h_{1} x_{1}, h_{2} x_{2}\right)=f t^{-1}\left(x_{1}, x_{2}\right)$ for all $x_{1}, x_{2} \in I$, $h_{1}, h_{2} \in G^{\prime}$, comp. [6], $\S 2$. Hence it may be regarded as a factor system of $F:=I / G^{\prime}$.

There is a representative $U$ of an irreducible complex projective representation of $F$ such that $R=A \otimes \inf _{F}^{I}(U)$. Since $F$ is abelian there is a subgroup $F_{0} \leqq F$ such that $U$ is induced by some function $\lambda: F_{0} \rightarrow C^{*}$ which satisfies $\lambda(a) \lambda(b)=f t^{-1}(a, b) \lambda(a b)$. $\lambda$ defines an element $\bar{\lambda} \in \operatorname{Hom}\left(F_{0}, C^{*} / K^{*}\right)$. We can find an element $\bar{\alpha} \epsilon$ Hom $\left(G / G^{\prime}, C^{*} / K^{*}\right)$ such that $\bar{\alpha} \mid F_{0}=\bar{\lambda}^{-1}$ and all values of the trivial factor system $\delta \alpha$ belong to $K^{*}$. Define $T^{\prime}:=\alpha \otimes T, f^{\prime}:=f \delta \alpha$. By Frobenius reciprocity (comp. [4], (1.12)) $T^{\prime}$ is induced by $A \otimes$ $\inf (\alpha \mid F \otimes U)$ and $\alpha \mid F \otimes U$ is induced by $\alpha \mid F_{0} \otimes \lambda$. All values of $\alpha \mid F_{0} \otimes \lambda$ belong to $K$. Hence $\alpha \mid F \otimes U$ is realizable in $K$ and then also $T^{\prime}$ is realizable in $K$. This shows that $P$ is realizable in $K$.

\section{REFERENCES}

1. J. L. Alperin and I. N. Kuo, The exponent and the projective representations of a finite group, Illinois J. Math., 11 (1967), 410-413.

2. A.J. Bayes, J. Kautsky and J.W. Wamsley, Computation in nilpotent groups (application), in: The Theory of Groups, LNM 372, Springer Verlag, Berlin-HeidelbergNew York, 1974, 82-89. 
3. B. Huppert, Endliche Gruppen I, Springer Verlag, Berlin-Heidelberg-New York, 1967.

4. H. Opolka, Zerfällungskörper verschränkter Gruppenalgebren, J. Algebra, 51 (1978), 579-596.

5. E. W. Read, The projective representations of the generalized symmetric group, J. Algebra, 46 (1977), 102-133.

6. W.F. Reynolds, Projective representations of finite groups in cyclotomic fields, Illinois J. Math., 9 (1965), 191-198.

Received December 7, 1979.

Mathematisches Institut

ROXELER STRASSE 64

D-4400 MÜnster, W. Germany 


\section{PACIFIC JOURNAL OF MATHEMATICS}

\section{EDITORS}

DONALD BABBITT (Managing Editor)

University of Galifornia

Los Angeles, California 90024

Hugo RossI

University of Utah

Salt Lake City, UT 84112

C. C. MOORE AND ANDREW OGG

University of California

Berkeley, CA 94720

\section{J. DugundjI}

Department of Mathematics University of Southern California

Los Angeles, California 90007

R. Finn AND J. Milgram

Stanford University

Stanford, California 94305

\section{ASSOCIATE EDITORS}

R. ARENS

E. F. BECKENBACH

B. H. NeUmanN

F. WOLF

K. YosHIDA

\section{SUPPORTING INSTITUTIONS}

UNIVERSITY OF ARIZONA

UNIVERSITY OF BRITISH COLUMBIA

CALIFORNIA INSTITUTE OF TECHNOLOGY

UNIVERSITY OF CALIFORNIA

MONTANA STATE UNIVERSITY

UNIVERSITY OF NEVADA, RENO

NEW MEXICO STATE UNIVERSITY

OREGON STATE UNIVERSITY
UNIVERSITY OF OREGON

UNIVERSITY OF SOUTHERN CALIFONIA

STANFORD UNIVERSITY

UNIVERSITY OF HAWAII

UNIVERSITY OF TOKYO

UNIVERSITY OF UTAH

WASHINGTON STATE UNIVERSITY

UNIVERSITY OF WASHINGTON 


\section{Pacific Journal of Mathematics}

\section{Vol. 94, No. 1 \\ May, 1981}

Willy Brandal, Conditions for being an FGC domain $\ldots \ldots \ldots \ldots \ldots \ldots \ldots$

Allan Calder and Frank Williams, Incompressibility of maps and the

homotopy invariance of Čech cohomology $\ldots \ldots \ldots \ldots \ldots \ldots \ldots \ldots \ldots$

Jacques Chaumat, Quelques propriétés du couple d'espaces vectoriels

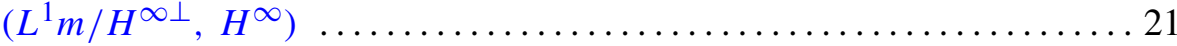

Manfred Droste and Rüdiger Göbel, Products of conjugate permutations . . 47

Jean Esterle, Rates of decrease of sequences of powers in commutative

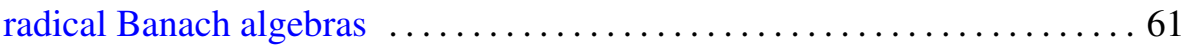

Allan Fryant, Ultraspherical expansions and pseudo analytic functions . . . 83

John Hannah, Homogenization of regular rings of bounded index. II . . . . . 107

Shigeru Haruki, On the theorem of S. Kakutani-M. Nagumo and J. L. Walsh

for the mean value property of harmonic and complex polynomials $\ldots .113$

Hugh M. Hilden, Representations of homology 3-spheres $\ldots \ldots \ldots \ldots \ldots 125$

Craig Huneke, A characterization of locally Macaulay completions . . . . . 131

Takesi Isiwata, Closed ultrafilters and realcompactness ................. 139

Joseph Weston Kitchen, Jr. and David A. Robbins, Tensor products of

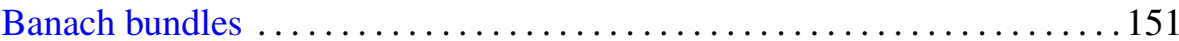

Allan J. Kroopnick, Note on bounded $L^{p}$-solutions of a generalized Liénard

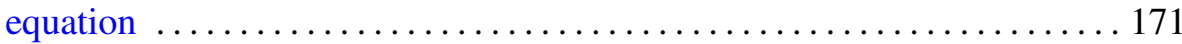

Ajay Kumar and Ajit Kaur Chilana, Spectral synthesis in products and quotients of hypergroups

Charles Livingston, Homology cobordisms of 3-manifolds, knot

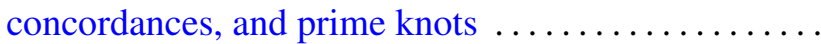

Hans Opolka, Projective representations of finite groups in cyclotomic fields

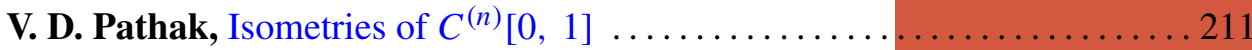

Mark Allan Pinsky, On the spectrum of Cartan-Hadamard manifolds . . . . . 2223

Judith Roitman, The number of automorphisms of an atomic Boolean

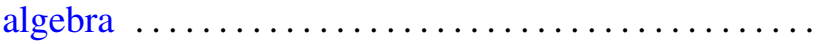

Kai Wang, Locally smooth torus group actions on integral cohomology complex projective spaces 